\title{
Reliability of a Cycle Sprint Test to Measure Lower Limb Muscle Power
}

Edmondston SJ ${ }^{1 *}$, Gibbons R ${ }^{1}$, Mackie KE', Haywood $Z^{1}$, Hince $D^{2}$ and Hurworth $M^{3}$

${ }^{1}$ Murdoch Centre for Orthopaedic Research, St J ohn of God Murdoch Hospital, Murdoch, WA 6150, Australia

${ }^{2}$ Institute for Health Research, University of Notre Dame, Australia

${ }^{3}$ Murdoch Orthopaedic Clinic, St J ohn of God Murdoch Hospital, Australia

*Corresponding author: Stephen Edmondston, Murdoch Centre for Orthopaedic Research, St J ohn of God Murdoch Hospital, 100 Murdoch Drive, Murdoch, WA 6150, Australia

Received: July 15, 2021; Accepted: August 31, 2021; Published: September 07, 2021

\begin{abstract}
This study examined the reliability of a cycle sprint test for measuring lower limb muscle power. Twenty asymptomatic volunteers completed the test on two occasions, with one week between test sessions. Participants sat on a stationary road bicycle with commercial power meters in the pedal cranks. Maximum and average muscle power was measured during three, 10-second sprint efforts. The test demonstrated excellent within- and between-day reliability for both maximum and average power measurement (ICC $=0.93$ to 0.97 ). The within-day Standard Error of Measurement (SEM) was between 25.9W (6.1\%) and 35.1W (8.5\%), and $24.8(6.5 \%)$ and $28.6 \mathrm{~W}(7.7 \%)$ for maximum and average power respectively. The between-day SEM was $34.3 \mathrm{~W}(7.8 \%)$ for maximum power and $26.4 \mathrm{~W}(7.1 \%)$ for average power. Reliability of the cycle sprint test has been established, along with thresholds for significant change. The cycle sprint test may have relevance in clinical populations to evaluate lower limb muscle power following injury, or to measure rehabilitation outcomes.
\end{abstract}

Keywords: Lower limb; Muscle power; Reliability; Functional outcomes; Cycle sprint test

\section{Introduction}

The impact of lower limb pathology and related functional deficit is commonly measured using patient-reported and physical function measures [1-3]. Since the loading environment of the knee joint is dependent on muscle function, it has been suggested that muscle function be evaluated in all patients with knee pathology $[4,5]$. Muscle function encompasses both strength and power. Muscle power is defined as "the product of dynamic muscular force and muscle contraction velocity", ([6] p3167) and has been found to be superior to muscle strength as a predictor of functional performance in healthy adults and patients with knee pathology $[7,8]$.

Muscle strength tests examine maximal exertion of a single muscle, often using open kinetic chain dynamometry $[9,10]$. However, closed kinetic chain measurement techniques may more closely reflect functional tasks. Aalund et al. [11] reported that leg press power was more closely associated with physical performance than quadriceps strength following knee arthroplasty. These findings highlight the potential importance of closed-chain evaluation of muscle power as an outcome measure in patients with knee pain or following intervention $[11,12]$. However, there has been limited documentation of reliable methods of muscle power evaluation that may be relevant in clinical populations, including older individuals.

Using a commercial power meter fitted to a standard road cycle, we have developed a cycle sprint test to assess lower limb muscle power. This test may support the evaluation of muscle power in clinical populations, particularly in monitoring outcomes of surgical treatment and rehabilitation. Before introducing this new method into clinical practice, there is a need to examine the acceptability and reliability in healthy participants, and to define thresholds for significant change. The aim of this study was to examine the reliability of a stationary cycle sprint test to assess lower limb muscle power in healthy individuals.

\section{Methods}

The study participants were healthy volunteers with no history of knee pain or symptoms of knee osteoarthritis. A minimum age of 18 years and adequate English were required to take part in the study. Participants were excluded if they had a history of previous lower limb surgery, unstable cardiovascular or respiratory conditions. Participants were provided information regarding the study and those who agreed to participate provided written consent. All procedures performed in this study involving human participants were in accordance with the ethical standards of the institutional committee that have approved them.

Muscle power was measured using the InfoCrank Power Meter (Verve Cycling, Australia). The InfoCrank has an absolute maximum error of $0.11 \mathrm{Nm}$ below $17 \mathrm{Nm}$ and $0.57 \%$ above $17 \mathrm{Nm}$, indicating that it is highly accurate within and between sessions [13]. The InfoCrank replaces the standard cranks on a bicycle and contains dual sided power meters that communicate with one another and function as one. This device directly measures torque applied to it via plastic deformation of the strain gauges under load and cadence (RPM). Power and cadence was collected at $256 \mathrm{~Hz}$ and transmitted to an ANT+ receiver, in this case an O_Synce Navi2coach bike computer (O_Synce, Germany). Data was analysed using a cycling analysis software program, Golden Cheetah for Windows (GoldenCheetah v3.4), and exported to Microsoft Excel for Windows to calculate the average and maximum power for each test.

A repeated measures design with seven days between tests was used. Testing was performed with the participant seated on a road bicycle (Pinarello, Italy) mounted on a stationary trainer (Revbox Erg, 


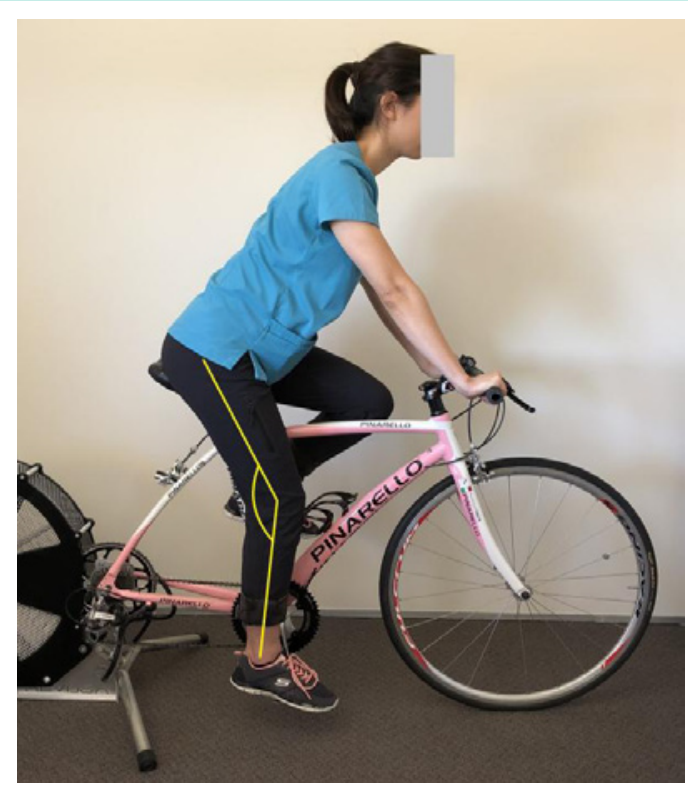

Figure 1: Bicycle fit position with downstroke knee angle of $25^{\circ}$ degrees.

New Zealand). The participants were fitted to the bicycle with a clipless pedal and standardized downstroke knee angle of 25 degrees (Figure 1) [14]. Participants were asked to perform a familiarization session of 1 minute on the bicycle to gain confidence and ensure comfort. For each participant, seat height and the self-selected gear setting were consistent on both testing days. Following the familiarization session, the participant was asked to perform a sprint of 10 seconds followed by a one-minute recovery. For the 'sprint' phase, the participant was instructed to 'pedal as hard and fast as you can'. The participants had either passive recovery (no pedalling) or active recovery (light pedalling) between each sprint based on their preference, and this was consistent between trials and within test days. The sprint/rest phases were repeated two more times. The testing for each participant was carried out at approximately the same time of day, to reduce the possible impact of circadian rhythm on test performance $[15,16]$.

\section{Statistical analysis}

Raw data was recorded at one reading per second. The main dependent variables were maximum power output and average power output (in Watts) generated during the 10-second sprint phases. For maximum power, both the single highest power reading (maximum power) and an average of the maximum for each sprint phase (mean maximum power) were investigated. For the within day analyses, these were calculated for each sprint phase. For the between day analyses, these were calculated across the sprint phases, to give the mean or maximum for the testing day. Data was analysed using Stata v15 (StataCorp LLC, United States of America) and IBM SPSS Statistics v24 (IBM Corp, Australia), and p $<0.05$ was considered statistically significant.

Reliability of the cycle sprint test protocol was investigated both within the testing day, and between testing days. Intra-Class Correlation Coefficients (ICCs) were calculated from the two way mixed models, considering participants as a random factor, time as a fixed factor, and using the absolute agreement definition for a single rater in order to maximise generalisability (ICC $(2,1))$. Differences in mean power output measures across time (day 1 to day 2 or across the 3 sprint phases within each day) were assessed using the $\mathrm{F}$ test from these models. The standard error of measurement $\left(\mathrm{SEM}=\mathrm{SD} \mathrm{V}^{*}(1-\right.$ ICC)), percent standard error of measurement $(\%$ SEM $=($ SEM $/$ mean $\left.)^{\star} 100\right)$ and minimal detectable change $\left(\mathrm{MDC}=1.96^{*} \sqrt{ } \mathrm{n}^{\star} \mathrm{SEM}\right.$ where $n=2$ for between day analyses and $n=3$ for within day analyses) were also calculated.

\section{Results}

Of the 20 participants, seven (35\%) were male and $13(65 \%)$ were female. Participant age ranged from 21 to 70 years (mean $=47$, $\mathrm{SD}=14$ years). Maximum and average power output were consistent both within and between test days. No significant differences were observed between sprint phases on day 1 or day 2 , or between day 1 and day 2 values.

The ICC scores were excellent for all within-day measures, ranging between 0.93 and 0.97 (Table 1). SEM values ranged from $24.8 \mathrm{~W}(6.5 \%)$ to $35.1 \mathrm{~W}(8.5 \%)$ and MDC values from $84.3 \mathrm{~W}(22.0 \%)$ to $119.0 \mathrm{~W}(28.7 \%)$. Maximum power has a measurement error of $35.1 \mathrm{~W}(8.5 \%)$, such that a change of $119 \mathrm{~W}(28.7 \%)$ is required for real change with $95 \%$ confidence. The between-day ICC scores were also excellent for maximum, mean maximum and average power (Table 2). Given the high degree of reliability between test days, we also assessed the reliability of the first sprint phase only between days. Under these conditions, the reliability was almost identical to that for the maximum and average power output calculated across the three sprint phases shown in Table 2 (maximum power output: ICC $=0.95$ ( $95 \%$ CI: 0.87 to 0.98 ), average power output: ICC $=0.94$ (95\% CI: 0.85 to 0.97$)$ ).

For every $1 \mathrm{~cm}$ increase in height, maximum power increased by $11.0 \mathrm{~W}$ (95\% CI: 4.16 to $17.8, \mathrm{p}=0.002)$; and for every $1 \mathrm{~kg}$ increase in weight, maximum power increased by $7.3 \mathrm{~W}$ (95\% CI: 2.66 to 12.0 , $\mathrm{p}=0.002$ ).

Table 1: Results for within-day analysis of maximum and average power output on the two testing days.

\begin{tabular}{|c|c|c|c|c|c|c|c|}
\hline & \multirow{3}{*}{ Day } & \multicolumn{3}{|c|}{ Test number } & \multirow{3}{*}{$\operatorname{ICC}(2,1)^{a}(95 \% \mathrm{Cl})$} & \multirow{3}{*}{ SEM $^{\mathrm{b}}(\%)$} & \multirow{3}{*}{ MDC $^{c}(\%)$} \\
\hline & & 1 & 2 & 3 & & & \\
\hline & & Mean (SD) & Mean (SD) & Mean (SD) & & & \\
\hline \multirow{2}{*}{ Maximum Power (W) } & 1 & $416.1(152.7)$ & $408.8(129.8)$ & $417.6(135.6)$ & 0.93 (0.87 to 0.97$)$ & $35.1(8.46)$ & $119.0(28.7)$ \\
\hline & 2 & $425.95(138.0)$ & 424.7 (140.8) & 432.7 (146.5) & 0.97 (0.93 to 0.99$)$ & $25.9(6.05)$ & $87.9(20.5)$ \\
\hline \multirow{2}{*}{ Average Power (W) } & 1 & 370.9 (124.9) & $368.6(115.2)$ & $375.4(120.7)$ & 0.94 (0.88 to 0.97$)$ & $28.6(7.70)$ & $97.1(36.1)$ \\
\hline & 2 & $383.6(121.4)$ & $384.0(121.8)$ & $382.5(121.6)$ & 0.96 (0.91 to 0.98$)$ & $24.8(6.48)$ & $84.3(22.0)$ \\
\hline
\end{tabular}

antraclass correlation coefficient using the absolute agreement definition; ' ${ }^{5}$ Standard error of measurement; ' ${ }^{M i n i m a l}$ detectable change. 
Table 2: Results for between-day analysis of maximum, mean maximum and average power outputs.

\begin{tabular}{|c|c|c|c|c|c|}
\hline & Day 1 & Day 2 & \multirow{2}{*}{$\operatorname{ICC}(2,1)^{a}(95 \% \mathrm{Cl})$} & \multirow{2}{*}{ SEM $^{\mathrm{b}}(\%)$} & \multirow{2}{*}{$\operatorname{MDC}^{\mathrm{c}}(\%)$} \\
\hline & Mean (SD) & Mean (SD) & & & \\
\hline Maximum Power (W) & $441.0(149.3)$ & $446.5(149.1)$ & 0.95 (0.87 to 0.98$)$ & $34.3(7.79)$ & $95.2(21.6)$ \\
\hline Mean Maximum Power (W) & $414.1(136.6)$ & $427.8(140.1)$ & 0.96 (0.90 to 0.98$)$ & $27.0(6.51)$ & $74.8(18.1)$ \\
\hline Average Power (W) & 371.6 (117.9) & $383.4(119.8)$ & 0.94 (0.85 to 0.97$)$ & $26.4(7.10)$ & $73.2(19.7)$ \\
\hline
\end{tabular}

antraclass correlation coefficient using the absolute agreement definition; ' ${ }^{b}$ Standard error of measurement; 'Minimal detectable change.

\section{Discussion}

The cycle sprint test demonstrated excellent within- and betweenday reliability, indicating that this protocol is a reliable measure of muscle power in healthy individuals. Importantly, taking the single maximum power reading was as reliable as using the mean maximum power or the average power over the three sprint phases. The high ICC scores are comparable to studies investigating reliability of open kinetic chain hand-held dynamometry $[9,10]$, indicating that the cycle sprint test is as reliable as clinical tests of muscle strength $[17,18]$.

Recommendations for minimizing measurement error in muscle function testing include using a familiarization period, three repeated trials and supervision by a trained therapist [19]. These factors were incorporated into the cycle sprint-test protocol employed in the present study. When investigating the reliability of new devices and protocols, it is important to identify the influence of a learning effect on the results. No learning effect was evident in this study, probably due to the closed-chain nature of the test, which has little demand on co-ordination or skill development requirement. The sprint period of 10 seconds limits the impact of cardiorespiratory fatigue on test performance [20]. The one-minute rest time between sprints is based on the recommendations for recovery between repeated lower limb muscle power tests [11].

Despite the easy set-up of this device and its use in healthy participants, patients with lower limb pain or osteoarthritis may have difficulty mounting or retaining balance on the static bicycle. The other significant limitation to completing the cycle sprint test in a patient population is limitation of knee flexion. At least 100 degrees knee flexion is required to complete the test with a symmetrical pedalling action, while remaining seated. Finally, the test requires some level of cardiovascular exertion, and achieving a true maximum power output requires a high level of motivation for the duration of the test. These factors should be considered when screening patients for suitability for this test, and when analyzing results of repeated tests over time.

\section{Conclusion}

In conclusion, this study has demonstrated excellent reliability of a cycle-based muscle power test in healthy participants using a commercial power meter. Thresholds for significant change have been established which will assist the interpretation of studies examining changes in lower limb muscle power over time. The utility of the cycle sprint test, as a measure of muscle function in patients with lower limb pathology, requires further evaluation in relevant clinical populations.

\section{References}

1. Murray DW FR, Rogers K, Pandit H, Beard DJ, Carr J, Dawson J. The use of the Oxford hip and knee scores. J Bone Joint Surg. 2007; 89: 1010-1014.

2. Roos EM RH, Lohmander LS, Ekdahl C, Beynnon BD. Knee injury and osteoarthritis outcome score (KOOS) - development of a self-administered outcome measure. JOSPT. 1998; 78: 88-96.

3. Ramkumar PN, Harris JD, Noble PC. Patient-reported outcome measures after total knee arthroplasty: a systematic review. Bone \& joint research. 2015; 4: 120-127.

4. Dreinhöfer KSG, Ewert T, Ebenbichler G, Gutenbrunner C, Kostanjsek N, Cieza A. ICF Core Sets for osteoarthritis. J Rehabil Med. 2004; 44: 75-80.

5. Maffiuletti NA. Assessment of hip and knee muscle function in orthopaedic practice and research. J Bone Joint Surg Am. 2010; 92: 220-229.

6. Reid KF PL, Harvey WF, Driban JB, Hau C, Fielding RA, Wang C. Muscle power is an independent determinant of pain and quality of life in knee osteoarthritis. Arthritis and Rheumatology. 2015; 67: 3166-3173.

7. Berger MJ MC, Chess DG, Goela A, Doherty TJ. Quadriceps neuromuscular function and self-reported functional ability in knee osteoarthritis. J Appl Physiol. 2012; 113: 255-262.

8. Reid KF, Fielding RA. Skeletal muscle power: a critical determinant of physical functioning in older adults. Exerc Sport Sci Rev. 2012; 40: 4-12.

9. Hartmann A KR, Murer K, de Bruin ED. Reproducibility of an isokinetic strength-testing protocol of the knee and ankle in older adults. Gerontology. 2009; 55: 259-268.

10. Koblbauer IF LY, Van Der Hulst ML, Neeter C, Engelbert RH, Poolman RW, Scholtes VA. Reliability of maximal isometric knee strength testing with modified hand-held dynamometry in patients awaiting total knee arthroplasty: useful in research and individual patient settings? A reliability study. BMC Musculoskeletal Disorders. 2011; 12: 249-258.

11. Aalund PK, Larsen K, Hansen TB, Bandholm T. Normalized knee-extension strength or leg-press power after fast-track total knee arthroplasty: which measure is most closely associated with performance-based and selfreported function? Arch Phys Med Rehabil. 2013; 94: 384-390.

12. Silva M, Shepherd EF, Jackson WO, Pratt JA, McClung CD, Schmalzried TP. Knee strength after total knee arthroplasty. J Arthroplasty. 2003; 18: 605-611.

13. Accuweigh. Breakaway Innovations (BAI) Verve Cycling Infocrank Statement of Accuracy. Perth. 2014.

14. Asplund C SPP. Knee pain and bicycling: fitting concepts for clinicians. Phys Sportsmed. 2004; 32: 23-30.

15. Lericollais R, Gauthier A, Bessot N, Davenne D. Diurnal evolution of cycling biomechanical parameters during a 60-s Wingate test. Scand J Med Sci Sports. 2011; 21: e106-114.

16. Souissi N, Driss T, Chamari K, Vandewalle H, Davenne D, Gam A, et al. Diurnal variation in Wingate test performances: influence of active warm-up. Chronobiol Int. 2010; 27: 640-652.

17. Stark T, Walker B, Phillips JK, Fejer R, Beck R. Hand-held Dynamometry Correlation with the Gold Standard Isokinetic Dynamometry: A Systematic Review. PM\&R. 2011; 3: 472-479.

18. Carpenter M, Carpenter R, Peel J, Zukley L. The reliability of isokinetic and isometric leg strength measures among individuals with symptoms of mild osteoarthritis. Journal of Sports Medicine and Physical Fitness. 2006; 46: 585-589. 
19. Gagnon D, Nadeau S, Gravel D, Robert J, Belanger D, Hilsenrath M. Reliability and validity of static knee strength measurements obtained with a chair-fixed dynamometer in subjects with hip or knee arthroplasty. Archives of physical medicine and rehabilitation. 2005; 86: 1998-2008.
20. Gastin PB. Energy System Interaction and Relative Contribution during Maximal Exercise. Sports Medicine. 2001; 31: 725-741. 